Isele, Patrick; Höke, Julia

\title{
Reflexionen über digitales Lernen mit dem Sphero SPRK+. Erfahrungen mit Studierenden in der "Lernwerkstatt ${ }^{3}$ "
}

Holub, Barbara [Hrsg.]; Himpsl-Gutermann, Klaus [Hrsg.]; Mittlböck, Katharina [Hrsg.]; Musilek-Hofer, Monika [Hrsg.]; Varelija-Gerber, Andrea [Hrsg.]; Grünberger, Nina [Hrsg.]: lern.medien.werk.statt. Hochschullernwerkstätten in der Digitalität. Bad Heilbrunn : Verlag Julius Klinkhardt 2021, S. 231-247. - (Lernen und Studieren in Lernwerkstätten)

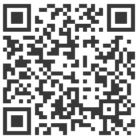

Quellenangabe/ Reference:

Isele, Patrick; Höke, Julia: Reflexionen über digitales Lernen mit dem Sphero SPRK+. Erfahrungen mit Studierenden in der "Lernwerkstatt ${ }^{3 "}$ - In: Holub, Barbara [Hrsg.]; Himpsl-Gutermann, Klaus [Hrsg.]; Mittlböck, Katharina [Hrsg.]; Musilek-Hofer, Monika [Hrsg.]; Varelija-Gerber, Andrea [Hrsg.]; Grünberger, Nina [Hrsg.]: lern.medien.werk.statt. Hochschullernwerkstätten in der Digitalität. Bad Heilbrunn : Verlag Julius Klinkhardt 2021, S. 231-247 - URN: urn:nbn:de:0111-pedocs-228232 - DOI: 10.25656/01:22823

https://nbn-resolving.org/urn:nbn:de:0111-pedocs-228232

https://doi.org/10.25656/01:22823

in Kooperation mit / in cooperation with:

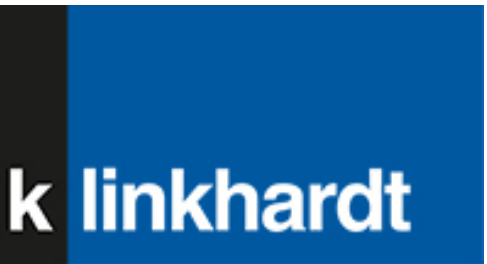

http://www.klinkhardt.de

\section{Nutzungsbedingungen}

Dieses Dokument steht unter folgender Creative Commons-Lizenz: http://creativecommons.org/licenses/by-nc-sa/4.0/deed.de - Sie dürfen das Werk bzw. den Inhalt unter folgenden Bedingungen vervielfältigen, verbreiten und öffentlich zugänglich machen sowie Abwandlungen und Bearbeitungen des Werkes bzw. Inhaltes anfertigen: Sie müssen den Namen des Autors/Rechteinhabers in der von ihm festgelegten Weise nennen. Dies Werk bzw. der Inhalt darf nicht für kommerzielle Zwecke verwendet werden. Die neu entstandenen Werke bzw. Inhalte dürfen nur unter Verwendung von Lizenzbedingungen weitergegeben werden, die mit denen dieses Lizenzvertrages identisch oder vergleichbar sind.

Mit der Verwendung dieses Dokuments erkennen Sie die Nutzungsbedingungen an.

\section{Terms of use}

This document is published under following Creative Commons-License: http://creativecommons.org/licenses/by-nc-sa/4.0/deed.en - You may copy, distribute and transmit, adapt or exhibit the work in the public and alter, transform or change this work as long as you attribute the work in the manner specified by the author or licensor. You are not allowed to make commercial use of the work. If you alter, transform, or change this work in any way, you may distribute the resulting work only under this or a comparable license.

By using this particular document, you accept the above-stated conditions of

\section{Kontakt / Contact:}

\section{peDOCs}

DIPF | Leibniz-Institut für Bildungsforschung und Bildungsinformation Informationszentrum (IZ) Bildung

E-Mail: pedocs@dipf.de

Internet: www.pedocs.de

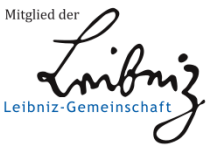




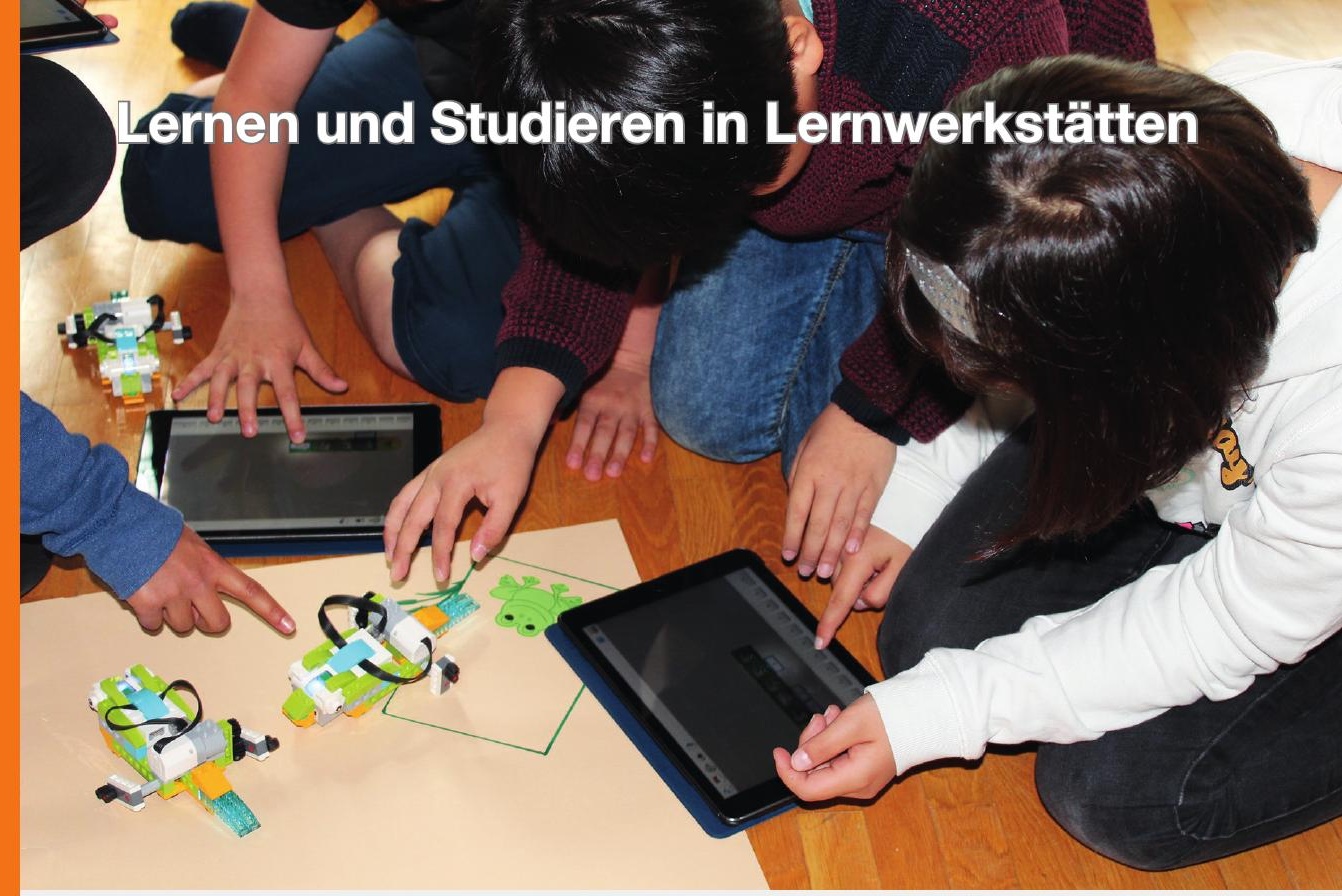

Barbara Holub / Klaus Himpsl-Gutermann Katharina Mittlböck / Monika Musilek-Hofer Andrea Varelija-Gerber / Nina Grünberger (Hrsg.)

\section{lern.medien.werk.statt}

Hochschullernwerkstätten in der Digitalität 
Barbara Holub Klaus Himpsl-Gutermann Katharina Mittlböck Monika Musilek-Hofer Andrea Varelija-Gerber

Nina Grünberger (Hrsg.)

\section{lern.medien.werk.statt}

Hochschullernwerkstätten in der Digitalität 
Dieser Titel wurde in das Programm des Verlages mittels eines Peer-Review-Verfahrens aufgenommen. Für weitere Informationen siehe www.klinkhardt.de.

Bibliografische Information der Deutschen Nationalbibliothek Die Deutsche Nationalbibliothek verzeichnet diese Publikation in der Deutschen Nationalbibliografie; detaillierte bibliografische Daten sind im Internet abrufbar über http://dnb.d-nb.de.

2021.ig. (C) by Julius Klinkhardt.

Coverfoto: (c) ZLI PH Wien.

Druck und Bindung: AZ Druck und Datentechnik, Kempten.

Printed in Germany 2021.

Gedruckt auf chlorfrei gebleichtem alterungsbeständigem Papier.

Die Publikation (mit Ausnahme aller Fotos, Grafiken und Abbildungen) ist veröffentlicht unter der Creative Commons-Lizenz: CC BY-NC-SA 4.0 International https://creativecommons.org/licenses/by-nc-sa/4.0/

ISBN 978-3-7815-5904-2 digital doi.org/10.35468/5904

ISBN 978-3-7815-2468-2 print 


\section{Inhaltsverzeichnis}

Barbara Holub, Klaus Himpsl-Gutermann, Katharina Mittlböck, Monika Musilek-Hofer, Andrea Varelija-Gerber und Nina Grünberger

Einführung in den Band 11

\section{Zur Konstituierung von Hochschullernwerkstätten}

Sandra Tänzer

Mut - Zumutung - Ermutigung. Oder wie man lernt, eine Hochschullernwerkstatt zu lieben

Markus Peschel, Hartmut Wedekind, Pascal Kihm und Mareike Kelkel

Hochschullernwerkstätten und Lernwerkstätten -

Verortung in didaktischen Diskursen

Dietlinde Rumpf und Corinna Schmude

Von der Herausforderung, die Vielfalt von Hochschullernwerkstätten

in einer Definition abzubilden

Pascal Kihm und Markus Peschel

„Komplexität wagen!“ - Methoden zur Beforschung von

offenen Lehr-Lern-Prozessen in Hochschullernwerkstätten 70

\section{Zum Spannungsverhältnis von Hochschullernwerkstätten und Digitalität}

Mark Weißhaupt, Ralf Schneider, Clemens Griesel und Agnes Pfrang

Digitale Erfahrung? Über das Lernen zwischen Instruktion und

(Ko-)Konstruktion

Stefan Brée, Lena S. Kaiser und Tanja Wittenberg

Lernwerkstatt als digitaler Erfahrungsort. Potenziale und

Herausforderungen für Lernwerkstätten als Orte

„offener multimedialer Produktionsästhetik“

\section{Ulrike Stadler-Altmann und Gerda Winkler}

Real \& virtuell, analog \& digital: Dimensionen einer Kooperation.

Multifunktionalität als Kennzeichen zukunftsfähiger Lernwerkstatt- und

Bibliothekskonzeption 


\section{Josef Buchner und Michael Kerres}

Lernwerkstattarbeit in der digital vernetzten Welt.

Die Perspektive der gestaltungsorientierten Mediendidaktik

Sabrina Tietjen und Silvia Thünemann

Forschungswerkstatt digital: ein modernes Lehr-Lernarrangement

für eine Digitalisierungsstrategie im Lehramt?

\section{Zu fach- und mediendidaktischen Perspektiven}

Johannes Mayer, Antonia Lemensieck, Maria Reinhardt und Karl Wollmann

Fachliche Perspektiven auf digitalisierungsbezogene Lernangebote

in der Ausbildung von Grundschullehrer*innen

Heike Hagelgans

Die Thematisierung digitaler Medien in den schulpraktischen Studien

Fachdidaktische Reflexionen von digitalen Medien für das Lernen im

Mathematikunterricht der Primarstufe

Jeanette Hoffmann, Katharina Egerer und Franziska Herrmann

Analoge Bilder - digitaler Film.

Möglichkeitsräume für literarästhetisches Lernen in

Hochschullernwerkstätten

Michael Rieseneder und Wolfgang Wagner

Erstes Programmieren mit Kindern über Handlungserfahrungen.

Das Konzept Activity-based-Coding

Patrick Isele und Julia Höke

Reflexionen über digitales Lernen mit dem Sphero SPRK+

Erfahrungen mit Studierenden in der „Lernwerkstatt ${ }^{3 “}$

Susanne Schumacher, Ulrike Stadler-Altmann und Enrico A. Emili

Piktogramme als Unterstützungsmedien.

Studien zu Effekten von Bilderbüchern mit zusätzlichen Symbolen im Kindergarten 


\section{Zu Aspekten, Perspektiven und Herausforderungen von Hochschullernwerkstätten}

Sabine Fischer und Max de Baey-Ernsten

Didaktische Perspektiven einer ästhetischen Werkstatt

Tanja Wittenberg und Lena S. Kaiser

„Ich war frustriert, ich bin kein Kind mehr“ - Erfahrungslernprozesse

mit verwendungs- und bedeutungsoffenem Material in

kindheitspädagogischen Hochschullernwerkstätten

Lisa Eßel und Laura Schlichting

Hochschullernwerkstätten im Spannungsfeld zwischen

Freiwilligkeit und Pflicht

Mareike Kelkel, Markus Peschel und Pascal Kihm

Potenziale der pädagogisch-didaktischen Öffnung in

Hochschullernwerkstätten

Barbara Holub und Sybille Roszner

Hochschullernwerkstatt - Ausgangspunkt für

persönlichkeitsorientierte Professionalisierung in der Ausbildung

für Lehrpersonen

Verzeichnis der Autor*innen 


\title{
Patrick Isele und Julia Höke
}

\section{Reflexionen über digitales Lernen mit dem Sphero SPRK+ \\ Erfahrungen mit Studierenden in der „Lernwerkstatt ${ }^{3 \%}$}

\begin{abstract}
Die Nutzung digitaler Medien wird mittlerweile nicht nur als Erscheinungsform des kindlichen Alltags betrachtet, sondern als neuer Bildungsbereich in den Bildungsplänen für Kindergärten und Kindertagesstätten (Kitas) festgeschrieben. Gleichzeitig wird die Mediennutzung junger Kinder kritisch diskutiert, und es werden negative Auswirkungen auf die kindliche Entwicklung befürchtet. Dabei treten bei Pädagog*innen bäufg Unsicherheiten auf, wenn es um die Nutzung von digitalen Geräten in den Einrichtungen und die Auseinandersetzung mit informatischen Themen und Fragestellungen geht. Reflexionsprozesse zur eigenen Haltung gegenüber dem Einsatz digitaler Medien im Elementarbereich vor dem Hintergrund konkreter Erfahrungen scheinen unerlässlich, um eine kompetente Auseinandersetzung zu ermöglichen. Im Rahmen des Seminars „Entwicklung im Spiel“ erprobten Studierende mit Kita-Kindern den Sphero SPRK+ als kugelförmigem Roboter, der sich mithilfe eines mobilen Endgerätes frei programmieren lässt. Das Lernwerkstattangebot zeichnete sich dadurch aus, dass sowohl Dozierende als auch Studierende und Kinder zum überwiegenden Teil über wenig Vorerfahrungen mit digital gesteuerten Robotern verfügten, hier also gemeinsam geteilte Denkprozesse im Sinne des Sustained Shared Thinking in besonderer Weise eröffnet wurden. Gerahmt wurde die praktische Auseinandersetzung durch Reflexionsbögen mit dem Ziel, vor, während und nach der Erprobung Haltungen und Deutungsmuster in Bezug auf das eigene sowie das kindliche Handeln festzuhalten und diese zu hinterfragen. Die Ergebnisse belegen, dass sich auch in der Studierendenschaft eine große Bandbreite von Interesse und Neugierde einerseits und Vorbehalten und Kritik andererseits zeigt.
\end{abstract}




\section{Hintergrund und Forschungsstand}

\subsection{Digitale Bildung: Bedeutung und Herausforderung für Kinder}

In unserer Informationsgesellschaft gelten die Fähigkeiten, digitale Geräte zu verstehen und zu bedienen, als Schlüsselkompetenzen für jetzige und folgende Generationen. In Bezug auf die digitale Bildung von Kindern wird für die Anwendungskompetenz von Informations- und Kommunikationstechnologien in diesem Zusammenhang häufig der Begriff der frühen "digital literacy" verwendet (vgl. z. B. Hasler Stiftung, 2013). Die Nutzungsfähigkeiten der Anwender*innen umfassen allerdings nur einen Teil der digitalen Bildung. Daneben wird einer kreativen Auseinandersetzung mit digitalen Medien hohe Bedeutung beigemessen. Es geht dabei darum, eigene Problemstellungen zu definieren, Lösungen zu entwickeln und diese auf ihren Erfolg/ihre Funktionalität zu überprüfen. Gemeint ist damit das sogenannte informatische Denken bzw. „Computational Thinking“, also die Entwicklung von technischem Verständnis, Logik, Kreativität, modellhaftem Lernen und Analyse komplexer Aufgabenstellungen in ihren Teilschritten (Bergner et al., 2018, S. 60). In diesem Zusammenhang wird diskutiert, digitale Bildungsmöglichkeiten möglichst früh in den Bildungssystemen zu etablieren.

Die Fähigkeit, unterschiedliche digitale Medien zu verstehen und anzuwenden, haben Kinder bereits im Alter von drei bis vier Jahren entwickelt (Palaiologou, 2016, S. 6). Automatisch werden digitale Kompetenzen jedoch nicht erworben, wie der vielzitierte Begriff der „digital natives“ (Palfrey et al., 2008) vermuten lässt. Dass allein das Hineingeboren werden in eine digitale Gesellschaft ausreicht, um mit digitalen Medien kompetent umgehen zu können, hat sich empirisch nicht bestätigt. So konnte beispielsweise in einer Studie mit Jugendlichen in Deutschland gezeigt werden, dass knapp 30\% der Teilnehmenden über unzureichende computer- und informationsbezogene Kompetenzen verfügen (Eickelmann, 2016).

Erste Schritte zum Programmieren können bereits in der Kita erlernt werden, wie diverse Erfahrungen der Stiftung "Haus der kleinen Forscher" zeigen. So bieten z. B. programmierbare Spielzeuge Erfahrungsraum für einen kreativen Umgang mit digitalen Medien. Sie ermöglichen den Kindern, das Grundkonzept der Programmierung als zentrale Kompetenz informatischen Denkens kennenzulernen, indem sie verstehen lernen, dass man z. B. Robotern kleinschrittige Handlungsanweisungen als Input geben muss, um sie steuern zu können (Bergner et al., 2018, S. 210).

Obwohl Kinder bereits in jungen Jahren in der Lage sind, auch informatische Konzepte zu erlernen, treten sie in erster Linie lediglich als Konsumenten digitaler Medien in Erscheinung, indem sie z. B. Medienangebote im Internet nutzen oder Computerspiele spielen. Eine konstruktive Nutzung von digitalen Medien, um neue und kreative Ergebnisse zu erzielen, stellt im Alltag von Kindern eher die 
Ausnahme dar. In einer wissenschaftlichen Untersuchung zur Arbeit der Stiftung "Haus der kleinen Forscher" sieht eine Expert*innengruppe genau hier Potenzial in Bezug auf die Bereitstellung von Angeboten durch Kitas und Schulen, um informatisches Denken bereits in jungen Jahren anzuregen und Kinder konstruktiv und kreativ die digitale Welt mitgestalten zu lassen (Bergner et al., 2018, S. 133). Dabei kommt den pädagogischen Fachkräften als Lernbegleiter*innen eine entscheidende Rolle zu.

\subsection{Digitale Bildung: Bedeutung und Herausforderung für pädagogische Fachkräfte}

Wie pädagogische Fachkräfte die damit verbundenen Herausforderungen angehen, hängt in hohem Maße von ihren Einstellungen und ihrer professionellen Kompetenz ab. Zunächst benötigen die pädagogischen Fachkräfte informatikdidaktische Kompetenzen, d. h. in erster Linie die Fähigkeit, Lernumgebungen zu erkennen bzw. zu schaffen, die das informatische Denken bei Kindern anregen und ihr Interesse für informatische Themen wecken und aufrechterhalten. Eine zentrale Voraussetzung für den Erwerb dieser fachdidaktischen Kompetenzen ist die eigene Einstellung zum Thema frühe informatische Bildung (Bergner et al., 2018, S. 36). Studien belegen allerdings, dass die Einstellungen gegenüber digitalen Medien bei pädagogischen Fachkräften ein sehr breites Spektrum aufweisen. So zeigte sich beispielsweise in einer Interviewstudie mit pädagogischen Fachkräften, dass ca. ein Drittel der Befragten digitale Medien im Kitaalltag ablehnten und die Aufgabe der Kita darin sah, Kinder von der Nutzung digitaler Medien abzuhalten (Six \& Gimmler, 2007). Der Aktionsrat Bildung (Vereinigung der Bayerischen Wirtschaft, 2018, S. 65) beschreibt unter Bezugnahme unterschiedlicher Fachliteratur drei zentrale Sorgen, die bezüglich des Konsums digitaler Medien angeführt werden. Diese könnten „a) Störungen der Aufmerksamkeit begünstigen, b) suchtartige und auf unmittelbare Bedürfnisbefriedigung zielende Nutzungsmuster begünstigen und c) entwicklungsförderliche andere Tätigkeiten (z. B. Sport) oder die Beschäftigung mit konventionellen Medien (z. B. Lesen eines Buches) verdrängen“.

In verschiedenen Studien wurde deutlich, dass sich die Einstellungen der pädagogischen Fachkräfte in mindestens zwei „Lager“ (Knauf, 2019) bzw. „HabitusTypen“ (Friedrichs-Liesenkötter, 2016) einteilen lassen. Die eine Seite betont vor allem die Risiken des Medienkonsums und betrachtet die Kita als Schutzraum, in dem die Kinder von digitalen Medien ferngehalten werden sollen. Die andere Seite sieht vor allem die Chancen durch Medien und betrachtet es dementsprechend als Aufgabe der Kita, Kindern im Rahmen der Medienerziehung den Umgang mit digitalen Medien zu ermöglichen und diesen pädagogisch zu begleiten. Süss (2018) identifiziert diesbezüglich insgesamt drei Grundhaltungen pädagogischer Fachkräfte. Die gegenüber digitalen Medien kritisch eingestellte Grundhal- 
tung bezeichnet er als kulturpessimistische, die auf die Chancen fokussierte als medieneuphorische Perspektive. Als dritte Perspektive beschreibt er eine kritischoptimistische Position, die die Nutzung und Wirkung von digitalen Medien von Rahmenbedingungen (z. B. Voraussetzungen der Anwender*innen, Qualität der medialen Inhalte, Intensität der Nutzung) abhängig macht. Aus dieser Perspektive kommt der Reflexion der Nutzung digitaler Angebote eine hohe Bedeutung zu. So sollte den Kindern ein positiver Umgang mit digitalen Medien ermöglicht, gleichzeitig sollten sie jedoch auch vor möglichen Gefahren geschützt werden. Die Herausforderung besteht demnach darin, ein dem Alter der Kinder entsprechendes individuelles und möglichst passgenaues Angebot an Erfahrungsmöglichkeiten zu bieten. Als zugrundeliegendes zentrales Ziel sämtlicher Bildungsbemühungen wird vorgeschlagen, digitale Souveränität anzustreben. Es geht dabei darum, dass Kinder lernen, digitale Medien eigenaktiv und selbstbestimmt anzuwenden und zu nutzen. Daher empfiehlt der Aktionsrat Bildung, digitale Medien bereits im Kitaalltag kreativ einzusetzen und in frühpädagogische Konzepte zu integrieren. Dementsprechend spielt die Vermittlung von digitalen Kompetenzen bereits im Kindesalter eine wichtige Rolle. Da das „Computational Thinking“von einer spielerischen und vorbehaltsfreien Auseinandersetzung auf Grundlage der Prinzipien des Ausprobierens, Experimentierens und Nachahmens ausgeht, können entsprechende Angebote auch schon Kindern im vor- und primarschulischen Bereich angeboten werden (Vereinigung der Bayerischen Wirtschaft, 2018, S. 87). Aus der Sicht der Kindheitspädagogik stellt sich dementsprechend einerseits die Frage, wie sich digitale Kompetenzen in der Praxis angemessen und kindgerecht vermitteln lassen. Andererseits ist ebenso ungeklärt, wie diese Kompetenzen in Ausbildung und Studium erworben werden können.

\subsection{Digitale Bildung: eine besondere Chance für Lernwerkstattarbeit?}

Lernwerkstattarbeit an Hochschulen ist eine besondere Form der Arbeit mit Studierenden, die sich über alternative Formen der Vermittlung und Aneignung und eine bestimmte Haltung gegenüber den Studierenden gestaltet. Die Kunst der Reflexion des eigenen Handelns als zentraler Punkt für professionelles pädagogisches Handeln soll in besonderer Weise erfahrbar werden. Zu dieser gehören die kritische Auseinandersetzung mit eigenen Vorstellungen, Grundorientierungen und Perspektiven, die kritische Auseinandersetzung mit Theorien, Studien, (bildungs-)politischen Instrumenten und Materialien (Gabriel et al., 2009). So wird zum Beispiel eine forschende Perspektive auf Material eingenommen; dieses wird entdeckt und untersucht, in gemeinsamer Auseinandersetzung erfahren und so Wissen und Sinn konstruiert. Ziel ist die reflexive Verknüpfung und Vertiefung von theoretischen Inhalten und die Entwicklung von Ideen für die Umsetzung in der Praxis, die sich mit Selbsterfahrungen über eigene Interessen, Haltungen und Bedürfnisse verknüpfen. So werden didaktische Materialien erprobt oder selbst 
entwickelt, das eigene pädagogische Agieren mit den Kommiliton*innen oder auch mit Kindern findet als Handeln „auf Probe“ in einem geschützten Setting statt. Lernwerkstattarbeit soll somit ko-konstruktive Lernprozesse und Lernen am gemeinsamen Gegenstand bzw. Sustained Shared Thinking (Siraj-Blatchford, 2009) ermöglichen. Sustained Shared Thinking meint dabei einen spezifischen Ansatz zur Interaktionsgestaltung mit Kindern, der sich durch einen offenen „Denkraum “ zwischen pädagogischen Fachkräften und Kindern auszeichnet. Die hier entstehenden Dialoge fokussieren ein gemeinsames Thema, Material oder Problem und versuchen, dieses gemeinsam zu erschließen oder zu lösen. Die gedankliche Auseinandersetzung führt zu einer Entwicklung und Erweiterung des Denkens (Sylva et al., 2004). Zentrale Gestaltungsmittel sind dabei offene Fragestrukturen und Diskussionen, die Erweiterung der kindlichen Gedanken und das Vorbildhandeln (Hopf, 2012). Lernwerkstattarbeit bietet an dieser Stelle die Chance, sich als Lernende*r in einem offenen „Denkraum" selbst zu erleben, eigene Forschungsfragen zu entwickeln und Lernwege zu erproben. Im Austausch mit Dozierenden und Kommiliton*innen entstehen offene Fragen und Diskussionen, die den eigenen Aneignungsprozess bereichern können. Durch die Reflexion der Erfahrungen entstehen (neue) Perspektiven für das eigene professionelle pädagogische Handeln.

Die "Lernwerkstatt " an der Katholischen Hochschule NRW, Abt. Paderborn wurde im Jahr 2018 eröffnet. Sie richtet sich an Studierende der drei Studiengänge Soziale Arbeit, Kindheitspädagogik und Religionspädagogik und bietet vielfältige Materialien zum Forschen, Lernen und Spielen. So finden sich sowohl Materialien zum Entdecken und Experimentieren, reformpädagogisches Material als auch klassisches analoges und digitales Spielmaterial in der Lernwerkstatt. Darüber hinaus stehen Bücher zum Forschenden Lernen ebenso wie verschiedene Bögen zu Beobachtung und Dokumentation zur Verfügung. Alle Materialien können während der wöchentlichen Öffnungszeiten frei und individuell erprobt werden. Darüber hinaus wird der Raum der Lernwerkstatt systematisch in Seminaren mit engem Praxisbezug genutzt. Die im Folgenden vorgestellte Forschungsarbeit ist im Kontext eines solchen Seminars entstanden.

\section{Methodischer Teil}

\subsection{Kontext des Seminars „Entwicklung im Spiel““}

Das Seminar „Entwicklung im Spiel“ gehört zum Modul 09 „Theorie und Praxis des kindlichen Spiels" des Bachelor-Studiengangs Kindheitspädagogik und ist hier eines von drei Seminaren, die im 3. bzw. 4. Fachsemester angeboten werden. Die beiden anderen Seminare heißen "Theorie des Spiels" und "Spielen und Spielmaterialien“. Das Modul hat insgesamt zum Ziel, den Studierenden Kompetenzen zu 
vermitteln, um Theorien des Spiels aus unterschiedlichen Disziplinen zu vergleichen, die Bedeutung des Spiels als menschliches Phänomen und als Medium und Ausdruck kindlicher Weltaneignung zu verstehen und die Bedeutung spezifischer Spiele und Spielmaterialien für das kindliche Lernen zu erfassen, diese situationsangemessen einzusetzen und deren Einsatz zu reflektieren. In der Durchführung der Seminare wird ein enger Theorie-Praxis-Bezug angestrebt, der durch eine enge Zusammenarbeit mit verschiedenen Kooperations-Kitas und dem Besuch von Kindern im Alter von 4 bis 6 Jahren in der Lernwerkstatt realisiert wird, in denen die Studierenden erworbenes Theoriewissen in die Praxis transferieren und sich selbst im Kontakt mit Kindern, in der Einführung von Spielmaterialien und in der Spielbegleitung erleben. Um Reflexionsprozesse des eigenen pädagogischen Handelns anzuregen, gibt es im Seminarverlauf sowohl individuelle als auch kollektive Reflexionsangebote (persönliches Reflexionsportfolio, Gruppendiskussionen, Reflexionsgespräche im Seminar).

\subsection{Integration des Themas Digitale Bildung im Seminar}

Im Seminar wurde ein Schwerpunkt auf das Thema Digitale Bildung gelegt, indem insgesamt vier Seminarsitzungen auf dieses Thema verwendet wurden.

Beim konkret erprobten Material handelt es sich um den Sphero SPRK+ („Schools, Parents, Robots, Kids") als kugelförmiger Roboter, der sich mithilfe eines unterstützten Computers oder mobilen Endgerätes frei programmieren lässt. Die Empfehlung des Herstellers für die Altersklasse liegt zwischen 3 und 12 Jahren. Für jüngere Altersgruppen stehen dabei einfache Bedienungsmöglichkeiten, wie z. B. das Programmieren von Bewegungen des Roboters durch Zeichnen am Tablet zur Verfügung, ältere Kinder können die Bewegungen und weitere Attribute des Roboters programmieren.

Das für die folgenden Analysen eingesetzte Datenmaterial besteht aus den drei Reflexionsbögen, die im Seminarverlauf zum Einsatz kamen. Alle Reflexionsbögen bestanden aus mehreren offen gestellten Fragen, zu denen die Studierenden ihre Gedanken notierten. Die Reflexionsbögen waren Teil des Reflexionsportfolios, welches die Studierenden über den gesamten Seminarverlauf führten. Zum Zweck der Datenauswertung wurden die Reflexionsbögen von den Studierenden pseudonymisiert, mit ihrem Einverständnis kopiert und die Originale an sie zurückgegeben.

1. Sitzung: Einführung in den Bildungsbereich Digitale Bildung allgemein

- Auseinandersetzung mit bildungspolitischen Zielen und Ansprüchen

- Vorstellung verschiedener Einsatzmöglichkeiten digitaler Medien in der Kita

- Bearbeitung des Reflexionsbogens 1, Reflexionsschwerpunkt: (nicht) vorhandene Vorerfahrungen mit digitalen Medien in der Kita, Haltung zu digitaler Bildung als (un)wichtiges Thema für die Kindheitspädagogik 
2. Sitzung: Vorstellung und erste Erfahrungen mit dem Sphero

- Vorstellung des Sphero, technische Informationen und Handhabung durch Mitarbeiter der IT der Katholischen Hochschule NRW, Abt. Paderborn

- Präsentation verschiedener Einsatzmöglichkeiten des Roboters

- Erprobung durch die Studierenden

- Entwicklung verschiedener Angebote für den Einsatz in der Kita

- Bearbeitung des Reflexionsbogens 2, Reflexionsschwerpunkt: Einstellung zum Sphero, Erwartungen bzgl. des Einsatzes mit Kindern

3. Sitzung: Erprobung des Sphero mit Kindern

- Teilung der Seminargruppe in vier Kleingruppen, drei Gruppen besuchen Kooperationseinrichtungen und eine Gruppe führt ihr Angebot in der "Lernwerkstatt" "durch

- Erprobung des Sphero in den verschiedenen Settings

- Bearbeitung des Reflexionsbogens 3, Reflexionsschwerpunkt: Reflexion der Erwartungen und der tatsächlichen Erfahrungen im Einsatz mit dem Sphero, Benennung von Chancen und Grenzen

4. Sitzung: Gemeinsame Reflexion der Erfahrungen

- Berichte aus den verschiedenen Gruppen zu Erwartungen, Ablauf und Erkenntnissen aus der Erprobung

- Reflexionsschwerpunkt im Plenum: Handhabung und Eignung des Sphero, Zusammenarbeit und Unterstützung mit den und durch die Kooperationseinrichtungen

- Rückmeldung an die IT der Katholischen Hochschule NRW, Abt. Paderborn

\subsection{Forschungsfragen}

1. Welche Einstellungen der Studierenden zum Thema Digitale Bildung werden deutlich?

2. Entstehen durch die persönlichen Erfahrungen mit dem Sphero und die Erprobung mit Kindern Irritationen der eigenen Einstellungen und Perspektivenwechsel?

3. Finden sich Hinweise auf Prozesse des Sustained Shared Thinking (SST) bzw. gemeinsamen forschenden Lernens in der Reflexion der eigenen Erfahrung im Seminar bei den Studierenden?

4. Finden sich bei den Studierenden Hinweise auf den Transfer der eigenen Erfahrungen bzgl. des SST bzw. des gemeinsamen forschenden Lernens auf die Gestaltung des Angebots mit den Kindern?

5. Verändern sich Begründungslogiken und Deutungsmuster in den Reflexionen der Studierenden zum Thema Digitale Bildung? 


\subsection{Sample und Auswertungsmethoden}

Die Gruppe der Studierenden bestand aus insgesamt 22 Personen, die alle weiblich und zwischen 18 und 25 Jahre alt waren. Die Reflexionsbögen 1 und 2 wurden jeweils von allen 22 Studierenden ausgefüllt. Der Reflexionsbogen 3 wurde im Anschluss der Durchführung von den 11 Studierenden vor Ort bearbeitet. Im Rahmen der Inhaltsanalyse wurden aus den Antworten der Studierenden zu jeder Frage Kategorien gebildet. Anschließend wurden die Antworten den Kategorien dichotom zugeordnet und in das Statistikprogramm SPSS überführt. So konnte quantitativ deutlich gemacht werden, wie viele Studierende Antworten gaben, die einer entsprechenden Kategorie zugeordnet werden konnten. Mithilfe einer deskriptiven Analyse der Mehrfachantwortsets konnten schließlich zu jeder Frage Prozentwerte der Nennung einzelner Kategorien berechnet werden, auf die bei der anschließenden Ergebnisdarstellung Bezug genommen wird.

Aufgrund der relativ kleinen Stichprobe können die Ergebnisse lediglich Hinweise auf die Einstellungen von Studierenden der Kindheitspädagogik gegenüber digitalen Angeboten für Vorschulkinder liefern. Um gesicherte Erkenntnisse über das Thema zu erlangen, müssten ähnliche Untersuchungen mit weit größeren Stichproben umgesetzt werden. Sämtliche nachfolgend angeführten Ergebnisse sind unter Berücksichtigung der Limitation durch diese kleine Stichprobe zu betrachten.

\section{Ergebnisse}

\subsection{Welche Einstellungen der Studierenden zum Thema Digitale Bildung werden deutlich?}

Die Einstellungen der Studierenden sind insgesamt sehr heterogen und bilden im Wesentlichen die drei genannten Grundeinstellungen nach Süss (2018) ab. So werden in einigen Äußerungen der Studierenden Ängste deutlich, die der kulturpessimistischen Perspektive zugeordnet werden können. Bei einem beträchtlichen Teil der Studierenden (40,9\%) zeigt sich eine gewisse Skepsis gegenüber digitaler Bildung in der Kita im Allgemeinen und dem Einsatz von Minirobotern im Besonderen. In diesem Zusammenhang werden Gefahren (z. B. einer möglichen Abhängigkeit) angeführt und die Kita als möglicher Schutzraum definiert $(18,2 \%)$, in dem digitale Medien von den Kindern ferngehalten werden sollten. Verknüpft mit der Sorge, dass Kinder durch digitale Medien abhängig werden könnten, wird häufig auch die Befürchtung geäußert, dass andere Entwicklungsbereiche verdrängt werden könnten („Ich bin der Meinung, dass die Kinder viel $z u$ früh abhängig werden und schnell dabei vergessen, etwas zu tun, was die anderen Kompetenzen fördern könnte“ (A); "Ich könnte mir vorstellen, dass die Kinder 
dann die Lust an nicht medialem Spielzeng verlieren würden "(Winnie Puh)). Nach der Durchführung des Angebots mit den Kindern bestätigt sich für 36,4\% der Studierenden der Eindruck, dass derartige Angebote mit digitalen Medien erst frühestens ab der Grundschule angeboten werden sollten. Neben einem „zu früh“ wird auch ein „zu viel“ an Kontakt mit digitalen Medien thematisiert, da die Kinder laut den Studierenden im Elternhaus bereits genug mit digitalen Medien in Kontakt kommen würden („Ich sehe den Einsatz digitaler Medien eher kritisch, weil die meisten Kinder meiner Meinung nach damit aufwachsen und es zu Hause lernen " (Sonne mit Gesicht), „Kinder spielen ähnliche Spiele auch am Smartphone - zu viel wenn es dann auch in der Kita gemacht wird" (Blume)). Den Kindern werden allerdings bereits weitreichende digitale Kompetenzen zugeschrieben („Ich denke, dass die Kinder den Sphero schneller und besser verstehen werden als wir" (Winnie Puh)). Gleichzeitig werden die eigenen Kompetenzen im Umgang mit dem Sphero geringer eingeschätzt als die Kompetenzen der Kinder (,Ich habe keine Erfahrungen und befürchte, dass die Kinder besser damit umgehen können als ich! Ich kann mir über den Einsatz noch nichts vorstellen "(Herz)). Deutlich werden zudem normative Vorstellungen darüber, was eigentlich als kindliche Stärke oder auch Ressource gesehen wird - Kinder, die den Studierenden als besonders kompetent beim Umgang mit dem Tablet auffallen, werden eher mit Sorge betrachtet. Dies wird besonders in folgendem Zitat deutlich: „Ein Kind war im Minispiel extrem geschickt (10.000 Punkte), was mit einem wissenden Blick der Erzieherinnen kommentiert wurde - ggf. wäre Digitale Bildung für dieses Kind zu viel aufgrund des Konsums im Elternhaus"(Wolke).

Die digitalen Kompetenzen der Kinder werden demnach nicht gewürdigt, sondern eher kritisch beurteilt. Um einem „zu früh “bzw. „zu viel“ der Nutzung digitaler Medien entgegenzuwirken, benennen mehr als ein Viertel der Studierenden (27,3\%) unterschiedliche Maßnahmen (bezogen auf Raum, Zeit oder Alter der Kinder), um den Einsatz digitaler Medien zu reglementieren bzw. zu kontrollieren. Diesen Maßnahmen liegt die Sorge zugrunde, dass die Kinder ansonsten möglicherweise gar nicht mehr aufhören können bzw. dass es Streit um die Spheros geben könnte, da mehr Kinder anwesend sein werden als Spheros vorhanden sind („Die Kinder wollen wahrscheinlich nur noch damit spielen“(Sternchen); „Ich denke der Sphero kann gut zum Spielen sein, jedoch für so viele Kinder wäre er denke ich nicht vorteilhaft, da es meiner Meinung nach Ärger geben könnte, wer jetzt spielen darf"(Herzchen)).

Auch die medieneuphorische Perspektive wird deutlich, indem die Bedeutsamkeit der digitalen Bildung hervorgehoben wird. So betonen fast zwei Drittel der Studierenden die zentral wichtige Bedeutung dieses Bildungsbereichs auch in Kindertageseinrichtungen (63,6\%). Dabei wird unter anderem Bezug genommen auf die aktuelle gesellschaftliche Entwicklung und die Bedeutung von Medien für den Bildungserfolg zukünftiger Generationen (,Die Bedeutung des Themenfelds 
"digitale Bildung" schätze ich als sehr wichtig ein, heutzutage ist dieser Schwerpunkt kaum wegzudenken, da die Kinder täglich mit digitalen Medien konfrontiert werden. Sie sollten lernen damit umgehen zu können" (Manfred oder auch Manni)). Interessanterweise wird ebenfalls deutlich, dass die Studierenden davon ausgehen, dass der Sphero für die Kinder neu sein und dementsprechend Begeisterung hervorrufen und ihre Aufmerksamkeit in den Bann ziehen wird („Ich denke, dass die Kinder davon begeistert sein werden. Es kann gut sein, dass die Kinder sich so gut wie die ganze Zeit (mit einigen Ausnahmen) damit beschäftigen werden" (Sonne mit Gesicht); „Kinder sind begeistert davon, da es sowas in Kitas nicht gibt - treffen auf Neues" (Sternchen)). Auch kompensatorische Effekte in Bezug auf soziale Ungleichheiten werden vereinzelt thematisiert („Den Einsatz digitaler Medien finde ich gut, da Kinder, welche ansonsten keinen Kontakt zu digitalen Medien haben, so die Möglichkeit haben, dieses kennenzulernen"(Weihnachtsbaum)).

Parallel dazu wird durch ein Abwägen der genannten Gefahren und Chancen von digitalen Angeboten für Kinder von den Studierenden auch die kritisch-optimistische Perspektive in den Blick genommen. Dabei wird die Chance betont, Kinder in der Kita auf den Umgang mit digitalen Medien vorbereiten zu können und ihnen eine gewisse Medienkompetenz zu vermitteln, so dass sie die damit verbundenen Gefahren abschätzen und ihnen entsprechend begegnen können („Da digitale Bildung die Kinder im Alltag immer begleiten, finde ich, dass das Themenfeld als sehr bedeutsam gilt. Im Internet können die Kinder auf viele Gefahren treffen. In der Kita würde ich digitale Bildung einbringen und den Umgang damit thematisieren. Besonders bei Fragen der Kinder würde ich digitale Medien (aber auch Bücher etc.) zur Klärung einbeziehen \& zeigen, dass die Medien nicht nur Vergnügen, sondern auch nützlich sind" (Dornröschen)). In diesem Zusammenhang wird von der Hälfte der Studierenden betont, wie wichtig dabei die Begleitung durch Erwachsene ist bzw. dass einer kompetenten Medienerziehung hohe Bedeutung zukommt. Knapp ein Viertel der Studierenden (22,7\%) betonte die Bedeutung einer gemeinsamen kritischen Reflexion solcher Medien mit den Kindern.

\subsection{Entstehen durch die persönlichen Erfahrungen mit dem Sphero und die Erprobung mit Kindern Irritationen der eigenen Einstellungen und Perspektivenwechsel?}

Nach der eigenen Auseinandersetzung mit dem Sphero beschreibt der Großteil der Studierenden die Erfahrung als ein positives Erlebnis (86,4\%) („Ich war begeistert, weil ich so etwas noch nie ausprobiert habe. Es hat Spaß gemacht, das Sphero kennenzulernen und damit zu spielen "(Rapunzel)). Dabei schätzen 81,8\% der Studierenden den Sphero als geeignet ein, um Kindern digitale Bildung nahezubringen. Mit Blick auf das bevorstehende Angebot mit Kindern wurde von mehr als zwei Dritteln der Studierenden Vorfreude geäußert $(68,2 \%)$ und auch in Bezug auf die 
Reaktion der Kinder auf den Sphero wurden von der Mehrheit der Studierenden positive Reaktionen erwartet $(57,1 \%)$.

Offensichtlich gab es bestimmte Vorstellungen der Studierenden über das digitale Angebot mit dem Sphero, die sich in der Selbsterfahrung jedoch nicht bestätigten („Ich hatte eine ganz andere Vorstellung von dem Roboter" (Rumpelstilzchen)). Im Gegenteil äußerten die Studierenden, dass sich ihre Skepsis im Laufe der Auseinandersetzung mit dem Sphero gewandelt hat („Ich war über die Vielfältigkeit positiv überrascht“(Stern); „Zu Beginn war ich sehr skeptisch. Nun bin ich ein wenig offener für dieses Thema" (Stern 2)). Gleichzeitig schätzten jedoch auch 22,7\% der Studierenden die Vor- und Nachteile des Sphero skeptisch ein. Für 13,6\% der Studierenden ist der Sphero für digitale Bildung nicht geeignet und die Kita wird von ebenfalls 13,6\% weiterhin als Schutzraum interpretiert, in dem digitale Medien, wenn überhaupt, nur reglementiert bzw. kontrolliert von den Kindern genutzt werden sollten $(9,1 \%)$. Von knapp einem Viertel der Studierenden wird erneut die gemeinsame kritische Reflexion im Umgang mit dem Sphero als besonders wichtig erachtet $(22,7 \%)$.

Im Anschluss an das Angebot mit den Kindern hat sich die Befürchtung, dass sich die Kinder nur noch mit dem Sphero beschäftigen würden und die Beschäftigung gar zu viel werden könnte, nicht bestätigt („Meine Erwartung, dass die Kinder viel Spaß haben, hat sich nicht immer bestätigt, da einige Kinder sagten, dass es für den Moment gut ist, aber sie es nicht immer im Kindergarten benötigen “(Herz); „Ich habe mich sehr wohl gefühlt, da ich später auch im Kindergarten arbeiten möchte. Allerdings waren die Kinder nicht so lange begeistert wie erwartet, sie waren nicht wirklich interessiert größtenteils" (Herzchen)). Fast die Hälfte der Studierenden zeigt sich überrascht, dass das Interesse der Kinder am Sphero bereits nach kurzer Zeit nachlässt (45,5\%) („Ich dachte, dass die Kinder noch mehr interessiert sind. “(Herz)). Dies wird von 18,2\% der Studierenden damit begründet, dass die Kinder offensichtlich (im Elternhaus) bereits (zu viel) Kontakt mit digitalen Medien haben und deshalb schnell das Interesse verlieren („Kinder nehmen Technik anders wahrnichts Besonderes"(Sonne)). Wiederum für 36,4\% der Studierenden bestätigt sich der Eindruck, dass derartige Angebote mit digitalen Medien wie dem Sphero für Kitakinder weniger geeignet sind und erst ab der Grundschule zum Einsatz kommen sollten („Ich hatte erwartet, dass das Sphero eher was für ältere Kinder geeignet ist und dies hat sich in der Durchführung auch bestätigt" (Rapunzel)). Die Sorge, dass es Streit wegen der geringen Anzahl an Spheros geben könnte, stellte sich als unbegründet heraus und wurde dementsprechend bei der Reflexion des Angebots nicht mehr thematisiert.

Andererseits wurde in der Reflexion des Angebots mit den Kindern von 36,4\% der Studierenden eine Offenheit der Kinder gegenüber dem Angebot beobachtet („Bin erstaunt, dass so eine kleine Kugel sie so begeistert hat und dass sie nicht gelangweilt wurden"(PJ Masks). 


\subsection{Finden sich bei den Studierenden Hinweise auf Prozesse des SST bzw. gemeinsamen forschenden Lernens in der Reflexion der eigenen Erfahrung im Seminar?}

In der Reflexion der eigenen Erprobung berichten 13,6\% der Studierenden explizit, dass sie sich den Roboter völlig anders vorgestellt haben, z. B. „Ich dachte, wir müsten mit einem "richtigen" Roboter arbeiten" (Rapunzel 1.0) oder "Ich dachte, wir haben einen Roboter, der sprechen, laufen etc. kann" (Dornröschen). Diese Irritation könnte darauf hindeuten, wie wichtig die persönliche Erfahrung zur Erfassung des gesamten Spektrums von digitaler Bildung ist, um die eigenen Vorstellungen zu erweitern. Die Einführung in die Arbeit mit dem Sphero erfolgte durch eine kurze Erklärung der Grundfunktionen und eine längere freie Erprobung in Kleingruppen. In der Beobachtung dieser Seminarphase wurde deutlich, dass die Studierenden sich bei der Bedienung abwechselten und gegenseitig unterstützten, Ideen entwickelten, was man ausprobieren könnte und sich gegen Ende der Phase gegenseitig Herausforderungen stellten (z. B. unter einem Stuhl hindurch oder besonders schnell über den Flur zu fahren; in die Fahrt Farbwechsel zu integrieren). Diese Erfahrungen lassen sich durchaus unter Aspekten des gemeinsamen forschenden Lernens beschreiben. In der Reflexion beschreiben 86,4\% der Studierenden die Erprobung als positives Erlebnis. Zudem sagen 27\%, dass sie die Bedienung des Sphero erst erlernen mussten, sie diesen also nicht intuitiv direkt bedienen und steuern konnten.

\subsection{Finden sich bei den Studierenden Hinweise auf den Transfer der eigenen Erfahrungen bzgl. des SST bzw. des gemeinsamen forschenden Lernens auf die Gestaltung des Angebots mit den Kindern?}

Dass die von den Studierenden selbst erlebte und beschriebene Lernerfahrung bei der Bedienung des Sphero auch für die Kinder relevant ist, wird von den Studierenden vereinzelt thematisiert (z. B. „Die Kinder sollten erstmal ein Gefühl für das Gerät entwickeln, bevor die einzelnen Spiele und Funktionen ausprobiert werden können"(WinniPooh)), allerdings nicht mit dem Blick darauf, wie diese in der Gestaltung des Angebots unterstützt und begleitet werden könnte. Ihre Hauptaufgabe sehen die Studierenden dagegen eher im Reglementieren und Kontrollieren der Spieldauer (z. B. „Die Spiele interessieren die Kinder wahrscheinlich am meisten, könnten sich lange damit beschäftigen bis Erzieher Stopp sagen", (Sternchen)) bzw. in der Organisation des Ablaufs. Besonders thematisiert wird von den Studierenden dabei die Sorge um Streit, die vor allem bei der Frage bzgl. der Befürchtungen von 45,5\% der Studierenden benannt wird, "wenn alle Kinder auf einmal wollen " (PJ Masks). Zur Gestaltung des Angebots berichtet die Hälfte der Studierenden, dass sie bereits konkrete Ideen für die Umsetzung des Angebots mit den Kindern haben, 27,3\% dagegen haben noch keine konkrete Idee. Bei der Betrachtung der 
von den Studierenden beschriebenen Ideen fällt auf, dass diese sich auf die konkrete Aktivität mit dem Sphero beziehen (Kreise, Zahlen, Slalom fahren, Labyrinth aufbauen, malen, etc.), hier also Aufgaben erdacht werden, was die Kinder mit dem Sphero tun sollen. Nur eine Studierende äußert sich gespannt, "wie die Kinder damit spielen werden und was für Ideen die Kinder entwickeln werden "(Stern 2). Der fehlende Wissens- und Erfahrungsvorsprung führt an dieser Stelle also nicht dazu, dass Perspektiven des gemeinsamen Entdeckens und Ausprobierens entstehen bzw. die eigene Erfahrung des Lernens und der Auseinandersetzung auf die Gestaltung des Angebots transferiert wird. Dieser fehlende Transfer zeigt sich auch in den Antworten zur Reflexion des Angebots nach der Erprobung mit den Kindern. 54\% der Studierenden sagen, dass ihre Aufgabe während des Angebots das "Erklären" und das "Helfen" gewesen sei, gefolgt von "Zeigen" (36,4\%) und „Anleiten“(27,3\%). Zudem spielt das positive Feedback eine wichtige Rolle, z. B. beim „Loben“ (36,4\%) und beim „Ermutigen“(27,3\%). Aufgabenbeschreibungen, die sich eher in die Richtung des SST deuten lassen, werden ebenfalls benannt, insgesamt jedoch in geringerer Häufigkeit. Darunter werden das "Beobachten" (36,4\%), das „Kommentieren“ (27,3\%), das „Begleiten“ (27,3\%) und die „Gespräche mit Kindern" $(18,2 \%)$ gefasst. Die meisten Studierenden äußern sich zufrieden über den Ablauf des Angebots (72,7\%). Gleichzeitig sind einige Studierende überrascht, dass die Kinder, anders als von ihnen erwartet, entweder ein eher nur kurzzeitiges $(45,5 \%)$ oder sogar kein Interesse $(9,1 \%)$ am Sphero hatten. Wie bereits berichtet, wurde das beobachtete fehlende Interesse von den Studierenden unterschiedlich begründet - allerdings immer bezogen auf die (nicht) vorhandenen Vorkenntnisse und Einstellungen der Kinder. Dass das wahrgenommene fehlende Interesse auch etwas mit der Umsetzung des Angebots zu tun haben könnte, wird von den Studierenden nur insoweit thematisiert, dass eine kleinere Gruppe bzw. mehr Spheros wünschenswert gewesen wären, um den Kindern besser oder mehr erklären zu können (z. B. Medimops). Von einer Studierenden wird dabei kritisch angemerkt, die „Kinder fuhren lieber durch die Gegend, vor allem die jüngeren Kinder brauchten viel Anweisung" (Herz). Dass evtl. größere eigene Freiräume und freies Ausprobieren dem Interesse der Kinder zuträglich sein könnten, wird von den Studierenden nicht thematisiert.

Zusammenfassend kann in Bezug auf Prozesse des SST gesagt werden, dass sich innerhalb der Reflexionsbögen kaum Hinweise auf gemeinsam geteilte Denkprozesse mit den Kindern finden lassen. Die Studierenden freuen sich zwar auf die Erprobung mit den Kindern und sind auch gespannt auf deren Reaktion; dass diese Reaktionen jedoch konkreten Einfluss auf die Durchführung des Angebots hätten und z. B. Fragen und Ideen der Kinder für die Studierenden handlungsleitend wären, lässt sich nicht feststellen. Auch in der Reflexion der Durchführung finden zwar Perspektivwechsel bei den Studierenden statt, diese sorgen jedoch nicht unbedingt für Reflexionsprozesse hinsichtlich der Angebotsgestaltung. Es 
zeigt sich beispielsweise, dass sich einige Erwartungen bzgl. des Angebots nicht bestätigen, wie z. B. die langanhaltende Begeisterung der Kinder für den Roboter und die damit verbundene Aufgabe, die Spieldauer zu reglementieren oder auch das befürchtete Chaos, wenn die Kinder sich beim Spielen abwechseln müssen. Dies könnte daran liegen, dass die Studierenden diese Befürchtungen in ihre Angebotsplanung direkt mit einbezogen haben und z. B. zu Beginn des Angebots bestimmte Regeln zur Spielreihenfolge und -dauer festgelegt und den Kindern kommuniziert haben. Dafür würde auch sprechen, dass sich die beschriebenen Angebotsstrukturen durch klare und eindeutige Aufgaben auszeichnen, die die Kinder mit dem Sphero bewältigen sollten, und weniger durch freies Ausprobieren und Experimentieren. Anhand dieser Hinweise erscheint fraglich, inwieweit bei der Angebotsgestaltung tatsächlich ein offener „Denkraum “ entsteht, in dem sich die Studierenden mit den Kindern gemeinsam auf den Weg machen, sich mit dem Sphero und seinen Möglichkeiten auseinanderzusetzen. Deutlich wird, dass der Transfer des eigenen Erlebens in der Auseinandersetzung mit dem Sphero (eigene Lernerfahrung, keine Vorerfahrungen, Bedeutung der freien Erprobung) auf die Angebotsgestaltung mit Kindern schwerfällt.

\subsection{Verändern sich Begründungslogiken und Deutungsmuster in den Reflexionen der Studierenden zum Thema Digitale Bildung?}

Über alle Befragungen hinweg zeigt sich, dass die Studierenden ihre Einstellungen zum Thema Digitale Bildung kaum fachlich begründen. Ganz ausdrücklich findet sich nur bei einem Reflexionsbogen ein Verweis auf einen fachlichen Bezug: „Den Einsatz digitaler Medien sehe ich sehr kritisch, da ich der Meinung bin, dass die Kinder heutzutage zu Hause schon viel Kontakt mit digitalen Medien haben und der Kitaalltag deshalb frei von digitalen Medien sein sollte, um wichtige andere Grundlagen zu erlernen wie z. B. Bauklötze aufeinander aufzubauen“, denn "damit sich die wichtigsten neuralen Schaltkreise im Hirn ausbauen, brauchen Kinder vor allem eins: eigene Körpererfahrung" Zitat Gerald Hüther - Hirnforscher" (Stern). Einerseits schildern Studierende die aus ihrer Sicht hohe Bedeutung der Digitalisierung und sind der Meinung, dass Kinder lernen müssen, sich hier zurechtzufinden, andererseits existieren Vorstellungen eines „zu viel“, vor allem verknüpft mit der Vorstellung, dass Kinder ohnehin zu Hause bereits viel digitalen Input bekommen. Eine bewusste Reflexion verschiedener Ebenen von „Konsumieren digitaler Medien "und „Erlernen digitaler Kompetenzen“"findet dabei nicht statt. Diese widersprüchlichen Positionen, die sich nicht nur innerhalb der Studierendengruppen, sondern auch innerhalb einzelner Reflexionen finden lassen, werden zumeist aufgelöst durch den Verweis auf die Bedeutung von „Medienerziehung“ (50,0\%). Wie diese allerdings gestaltet sein soll, bleibt offen bzw. wird auf einen späteren Zeitpunkt in der Bildungsbiografie der Kinder verschoben, vor allem auf die Grundschulzeit. Deutlich werden dagegen starke normative Vorstellungen von "guter Kindheit", 
wenn Studierende darauf verweisen, welche Themen sie anstelle digitaler Bildung für Kinder im Kitaalter wichtiger finden. Genannt werden hier vor allem Naturerfahrungen, z. B. "In diesem Alter (Kitalter) finde ich es wichtiger, mit der Natur in Berührung zu kommen. Themen wie Achtsamkeit, Naturschutz, gesunde Ernährung halte ich für sinnvoller" (Stern 2), aber auch Bücher und die Bedeutung sozialer Interaktionen. Über den Reflexionszeitraum hinweg finden sich diesbezüglich keine Relativierungen, z. B. dass das Auseinandersetzen mit dem Sphero auch soziale Interaktion anregen kann. Zusammenfassend lässt sich sagen, dass zumindest in dem angebotenen Seminarformat Potenzial für die Irritationen von Vorannahmen und Deutungsmustern vorhanden ist und sich diese teilweise auch zeigen, diese Irritationen und Widersprüche jedoch an vielen Stellen auch in der Gesamtgruppe evtl. noch bewusster hätten reflektiert werden müssen.

\section{Fazit}

Die Erkenntnisse des Lernwerkstattangebots zum Thema Digitale Bildung lassen sich auf verschiedenen Ebenen reflektieren. Hinsichtlich der didaktischen Gestaltung muss sicherlich kritisch hinterfragt werden, ob für die Auseinandersetzung, Erprobung und Reflexion innerhalb des Seminars ausreichend Zeit zur Verfügung gestanden hat. Die Integration des Themas in das Seminar „Entwicklung im Spiel", in dem darüber hinaus noch vielfältige andere Themen bearbeitet werden, schränkte das Zeitbudget deutlich ein. Zudem wird in den Ergebnissen der Reflexionsbögen deutlich, dass individuelle Reflexionen oftmals begrenzt bleiben und kontinuierlich durch kollektive Reflexionsangebote ergänzt werden sollten. Grundsätzlich deuten die Erkenntnisse darauf hin, dass es sich lohnen würde, ein eigenes Seminar Digitale Bildung anzubieten. In der aktuellen Form bleibt das Lernwerkstattangebot hinter den Erwartungen zurück.

Bezüglich der forschungsmethodischen Reflexion wäre grundsätzlich sicherlich eine größere Stichprobe wünschenswert gewesen, oder zumindest eine konstante Beteiligung über alle drei Erhebungszeitpunkte hinweg. Aufgrund der kleinen Kohortengröße des genannten kindheitspädagogischen Studiengangs war eine größere Stichprobe nicht realisierbar. Um Veränderungen während der drei Erhebungszeitpunkte deutlicher zu machen, hätten die Reflexionsbögen kongruenter aufgebaut werden können. Dazu wäre es sinnvoll gewesen, die Fragen kürzer und prägnanter zu formulieren sowie zu allen drei Erhebungszeitpunkten vergleichbare Fragen zu stellen.

In Bezug auf die Einstellungen der Studierenden zur digitalen Bildung in kindheitspädagogischen Settings zeigten sich unterschiedliche Aspekte der drei Grundeinstellungen nach Süss (2018). Die Perspektiven der Studierenden zeichnen sich 
jedoch nicht dadurch aus, dass ihre Äußerungen immer klar einer Grundeinstellung zugeordnet werden können. So benennen die Studierenden häufig jeweils gleichermaßen kulturpessimistische und medieneuphorische Aspekte. In den Äußerungen der Studierenden werden gewisse Spannungsfelder deutlich, die darauf hindeuten, dass die Studierenden dem Thema Digitale Bildung in Kitas insgesamt eher ambivalent gegenüberstehen. Einerseits wird eine grundsätzliche Offenheit gegenüber dem Thema signalisiert, andererseits wird auch eine gewisse Grundskepsis deutlich. Dies hängt womöglich damit zusammen, dass die Bedeutung der digitalen Bildung von allen Studierenden mehr oder weniger deutlich betont wird, gleichzeitig wird immer wieder auf Gefahren hingewiesen und die Kita als Schutzraum deklariert, in dem Kinder vor einem „zu viel“ an digitalem Medienkonsum geschützt werden müssen. Die Rolle der Erwachsenen und ihre Verantwortung für eine kompetente Medienerziehung in der Begleitung von Kindern wird häufig als sehr wichtig erachtet. Wie eine kompetente Medienerziehung in der Praxis von Kitas aussehen könnte, bleibt allerdings unklar. Die Betonung von Maßnahmen zur Einschränkung der Mediennutzung könnte dafür sprechen, dass das Kontrollieren und Reglementieren als Teil einer kompetenten Medienerziehung verstanden wird. Andererseits wird aber auch betont, dass die gemeinsame kritische Reflexion von digitalen Medien für Kinder von zentraler Bedeutung ist, damit sie Medienkompetenz erwerben können.

Eine kritisch-optimistische Position, wie Süss (2018) sie beschreibt, kann dazu beitragen, einerseits die den digitalen Medien zugrundeliegende Chancen aufzugreifen und andererseits mögliche Gefahren im Blick zu behalten, also Kinder so zu unterstützen, dass sie im Sinne der digitalen Souveränität in der Lage sind, sich selbstbestimmt und verantwortungsvoll den Herausforderungen unserer zunehmend digitaler werdenden Gesellschaft zu stellen.

Abschließend ist kritisch anzumerken, dass bzgl. der Reflexion des Transfers von durch Lernwerkstattangebote entwickelten Kompetenzen in die Praxis deutlich wird, dass dies in der vorgestellten Studie nur sehr eingeschränkt geschehen ist. Die Gründe dafür sind sicherlich vielfältig und liegen zum einen in der didaktischen Gestaltung, bei der die Planung der Angebote innerhalb der Kleingruppen erfolgte und nicht mehr gemeinsam im Seminar diskutiert wurde. Hier hätten Fragen und Rückmeldungen dazu führen können, dass die Angebotsplanung und -durchführung weniger erwachsenenzentriert und stärker sensibilisiert auf die Kindesinteressen hätte erfolgen können. Zum anderen spielen gruppendynamische Effekte unter den Studierenden sicherlich auch eine Rolle.

Insgesamt wird aber auch deutlich, dass dieser Transfer von Kompetenzen in die Praxis bislang im Diskurs um Lernwerkstatt und Lernwerkstattarbeit kaum in den Blick genommen wird und sich hier ein großes Potenzial für zukünftige Untersuchungen auch jenseits des Themenfelds Digitale Bildung zeigt. 


\section{Literatur}

Bergner, N., Köster, H., Magenheim, J., Müller, K., Romeike, R., Schroeder, U. \& Schulte, C. (Hg.) (2018). Wissenschaftliche Untersuchungen zur Arbeit der Stiftung „Haus der kleinen Forscher": Band 9. Frühe informatische Bildung - Ziele und Gelingensbedingungen für den Elementar-und Primarbereich. Verlag Barbara Budrich.

Eickelmann, B. (2016). Bildungsgerechtigkeit 4.0. https://www.boell.de/sites/default/files/uploads/ 2015/04/2015-04-eickelmann_-_bildungsgerechtigkeit-4.0.pdf

Friedrichs-Liesenkötter, H. (2016). Medienerziehung in Kindertagesstätten. Habitusformationen angehender ErzieherInnen. Medienbildung und Gesellschaft: Band 34. Springer VS. https://doi. org/10.1007/978-3-658-12307-9

Gabriel, E., Gunzenreiner, J., Hagstedt, H., Hangartner, W., Kieweg, U., Krauth, I. M., Munk, W., Rangosch-Schneck, E., Speck-Hamdan, A. \& Wedekind, H. (2009). Positionspapier des Verbundes europäischer Lernwerkstätten (VeLW) e.V. zu Qualitätsmerkmalen von Lernwerkstätten und Lernwerkstattarbeit. https://www.forschendes-lernen.net/files/eightytwenty/materialien/VeLW-Broschuere.pdf

Hasler Stiftung (2013). Informatik im Lehrplan 21. https://pdfdokument.com/queue/informatik-imlehrplan-21-fit-in-it_59e57d461723dd731db97379.html

Hopf, M. (2012). Sustained Shared Thinking im frühen naturwissenschaftlich-technischen Lernen. Zugl.: Wuppertal, Bergische Univ., Diss., 2011. Internationale Hochschulschriften: Bd. 572. Waxmann.

Knauf, H. (2019). Digitalisierung in Kindertageseinrichtungen. Ergebnisse einer Fragebogenerhebung zum aktuellen Stand der Nutzung digitaler Medien. Bielefeld Working Paper 3. https://www.pedocs.de/ volltexte/2019/17999/pdf/Knauf_2019_Digitalisierung_in_Kindertageseinrichtungen.pdf

Palaiologou, I. (2016). Children under five and digital technologies: implications for early years pedagogy. European Early Childhood Education Research Journal 24(1), 5-24. https://doi.org/10.1080 /1350293X.2014.929876

Palfrey, J. G., Gasser, U. \& Reinhart, F. (2008). Generation Internet: Die Digital Natives: wie sie leben, was sie denken, wie sie arbeiten. Hanser. http://deposit.d-nb.de/cgi-bin/dokserv?id=3099219 \&prov=M\&dok_var=1\&dok_ext=htm

Siraj-Blatchford, I. (2009). Conceptualising progression in the pedagogy of play and sustained shared thinking in early childhood education: A Vygotskian perspective. Education and Child Psychology 26(2), 77-89.

Six, U. \& Gimmler, R. (2007). Die Förderung von Medienkompetenz im Kindergarten: Eine empirische Studie zu Bedingungen und Handlungsformen der Medienerziehung. Schriftenreihe Medienforschung der Landesanstalt für Medien Nordrhein-Westfalen: Bd. 57. Vistas.

Süss, D. (2018). Mediensozialisation: Aufwachsen in mediatisierten Lebenswelten. In D. Süss, C. Lampert \& C. W. Trültzsch-Wijnen (Hg.), Studienbücher zur Kommunikations- und Medienwissenschaft. Medienpädagogik (3. Aufl.). Springer VS.

Sylva, K., Melhuish, E., Sammons, P., Siraj-Blatchford, I. \& Taggart, B. (2004). The Effective Provision of Pre-school Education (EPPE) Project: Findings from pre-school to end of key stage 1. University of Wollongong. http://ro.uow.edu.au/sspapers/2156

Vereinigung der Bayerischen Wirtschaft (Hg.)(2018). Digitale Souveränität und Bildung. Waxmann. 